Sem cl assi cal wave functi on of the peri odi $c$ Toda I at ti ce

\begin{tabular}{|l|l|}
\hline 著者 & Nat suyana Aki hi ko \\
\hline $\begin{array}{l}\text { j our nal or } \\
\text { publ i cat i on ti t l e }\end{array}$ & Physi cs Let ter s A \\
\hline vol une & 177 \\
\hline nunber & 6 \\
\hline page range & $415-420$ \\
\hline year & 1993 06- 28 \\
\hline 出版者 & El sevi er \\
\hline 権利 & $\begin{array}{l}\text { (c) } 1993 \text { Publ i shed by El sevi er Sci ence B. V. Al I } \\
\text { ri ght s reser ved. }\end{array}$ \\
\hline URL & ht t p: //hdl . handl e. net /10297/619 \\
\hline
\end{tabular}




\title{
Semiclassical wave function of the periodic Toda lattice
}

\author{
A. Matsuyama \\ Department of Physics, Faculty of Liberal Arts, \\ Shizuoka University, 836 Ohya, Shizuoka 422, Japan
}

\begin{abstract}
:
The semiclassical wave function of the periodic Toda lattice is constructed by the Keller's method. It is shown that the integer quantum numbers have a close relationship to a structure of the invariant torus of the classical motion.
\end{abstract}


$æ$

The Toda lattice is an integrable nonlinear dynamical system and has been studied extensively over two decades [1]. For the $N$-particle periodic Toda lattice, there are $N$ constants of motion and they define the $N$-dimensional invariant torus in the $2 N$ dimensional phase space. Once the invariant torus is known, it is possible to construct the semiclassical wave function on the torus according to the Keller's method [2]. However, no explicit integrating variables have been found to separate coordinates and, thus, it is very difficult to visualize the explicit structure of the invariant torus. The quantum version of the periodic Toda lattice has been studied [3-9], and in Ref.[9], we have carried out systematic investigation of the periodic Toda lattice and numerical calculation was done for $N=3,4,5$ and 6 particle Toda lattices. We have found that the semiclassical EBK quantization is a good approximation and there exists a close relationship between the integer quantum numbers and the symmetry of the eigenfunction. The purpose of this Letter is to explore this relationship in more detail and point out that it is based on the structure of the invariant torus.

Let us briefly review the $N$-particle periodic Toda lattice. The Hamiltonian is given in a dimensionless form as

$$
H=\frac{1}{2} \sum_{i} p_{i}^{2}+\sum_{i} \exp \left(q_{i}-q_{i+1}\right)
$$

where we set $q_{N+1}=q_{1}$. The classical equations of motion can be written in a Lax form

$$
\frac{d L}{d t}=[B, L]
$$

where

$$
L=\left(\begin{array}{ccccc}
b_{1} & a_{1} & & & a_{N} \\
a_{1} & b_{2} & a_{2} & 0 & \\
& \ldots & \ldots & & \\
& 0 & \ldots & \ldots & \\
& a_{N-2} & b_{N-1} & a_{N-1} \\
a_{N} & & & a_{N-1} & b_{N}
\end{array}\right), B=\left(\begin{array}{ccccc}
0 & -a_{1} & & & a_{N} \\
a_{1} & 0 & -a_{2} & 0 & \\
& \ldots & \ldots & & \\
& & \ldots & \ldots & \\
& 0 & a_{N-2} & 0 & -a_{N-1} \\
-a_{N} & & & a_{N-1} & 0
\end{array}\right)
$$

and $a_{n}=\frac{1}{2} \exp \left(\left(q_{n}-q_{n+1}\right) / 2\right), b_{n}=\frac{1}{2} p_{n}$. Therefore the eigenvalues of the matrix $L$ are constants of motion and, thus, the coefficients of the characteristic polynomial of the 
matrix $L$ are also constants of motion, which are defined as follows ( $I$ stands for the $N \times N$ identity matrix.)

$$
\operatorname{det}(2 \lambda I-2 L)=\sum_{i} A_{i}(2 \lambda)^{N-i}-2
$$

$\left(A_{0}=1\right)$ The conserved quantities $A_{i}$ are in involution with the Hamiltonian, $\left[H, A_{i}\right]=0$, and also mutually in involution $\left[A_{i}, A_{j}\right]=0$. (note that $H=\frac{1}{2} A_{1}^{2}-A_{2}$ )

In quantum mechanics, the Hamiltonian and the conserved quantities are operators and are given by the canonical quantization, i.e. the momentum $p_{i}$ is replaced by the operator $-i \hbar \frac{\partial}{\partial q_{i}}$. Since we have used three physical parameters ( the mass of each particle, the strength and the range of the potential) as units, Plank's constant $\hbar$ cannot be scaled out. Although $\hbar$ is basically arbitrary, we set $\hbar=1$ in the follwing arguments. We are interested in symmetric properties of the semiclassical wave function and they do not depend on the size of $\hbar$.

In Ref.[9], we have shown that the Hamiltonian has a symmetry of the dihedral group $\mathrm{D}_{N}$ and the simultaneous eigenfunctions of the Hamiltonian $H$ and the conserved quantities $A_{i}$ are classified according to the irreducible representation of the $\mathrm{D}_{N}$ group. The dihedral group $\mathrm{D}_{N}$ consists of two generators $s$ and $t$ satisfying $s^{N}=1, t^{2}=1, t s t^{-1}=s^{-1}$. These operators are defined to shift the coordinates as

$$
\begin{aligned}
& s\left(q_{1}, q_{2}, \cdots, q_{N}\right)=\left(q_{2}, q_{3}, \cdots, q_{N}, q_{1}\right) \\
& t\left(q_{1}, q_{2}, \cdots, q_{N}\right)=\left(-q_{N},-q_{N-1}, \cdots,-q_{2},-q_{1}\right)
\end{aligned}
$$

In a matrix representation, $s$ and $t$ are orthogonal matrices, i.e. ${ }^{T} s s={ }^{T} t t=1$ where $T$ denotes a transposition. For $N=$ odd $(N=2 m+1)$, the $\mathrm{D}_{N}$ group has two 1-dimensional representations $\left(A_{1}, A_{2}\right)$ and $m$ 2-dimensional representations $E_{k}(1 \leq k \leq m)$, while it has another two 1-dimensional representations $\left(B_{1}, B_{2}\right)$ for $N=$ even $(N=2 m+2)$. We will call $A_{1} / A_{2}, B_{1} / B_{2}$ and $E_{k}$ representations as $A$-, $B$ - and $E$-symmetries respectively, since they come out in a pair. The eigenfunctions and the matrix elements of the conserved quantities $A_{i}$ are summaried in Table 1 . One should note that the eigenstates generally 
degenerate in two-fold. These eigenfunctions are transformed as follows

$$
\begin{aligned}
& s \phi(\boldsymbol{q})=\phi(s \boldsymbol{q})=\zeta^{l} \phi(\boldsymbol{q}) \\
& t \phi(\boldsymbol{q})=\phi(t \boldsymbol{q})=\phi(\boldsymbol{q})^{*}
\end{aligned}
$$

where $\zeta=\exp (2 \pi i / N)$ and $l=0, k$ for the $A-, E_{k}$-symmetries respectively. In the case of $N=$ even, $l=N / 2$ for the $B$-symmetry should be added.

The semiclassical quantization can be carried out by the EBK formulation. Since the Toda lattice is integrable, the Hamiltonian can be expressed in terms of the actions $I_{i}$ by a canonical transformation, and the semiclassical energy is given by the substitutions $I_{i} \rightarrow\left(n_{i}+\frac{1}{2}\right) h$, where $n_{i}$ is an integer quantum number and the Maslov index is 2 . In order to obtain action-angle variables $\left(I_{i}, \varphi_{i}\right)$, it is known that another canonical conjugate variables $\left(\nu_{i}, \mu_{i}\right)$ are useful since the action $I_{i}$ is expressed as an integral over one period of $\mu_{i}$ variable

$$
I_{i}=\oint \nu_{i}\left(\mu_{i}\right) d \mu_{i}
$$

The auxiliary spectra $\mu_{i}$ are defined by the eigenvalues of the reduced matrix $L^{*}$ which is the $(N-1) \times(N-1)$ matrix given by removing the first row and the first column from the matrix $L$. They are confined in $N-1$ intervals satisfying $|\Delta(\mu)| \geq 2$ (they are labeled as $\left.\mu_{1}<\mu_{2}<\cdots<\mu_{N-1}\right)$. The function $\Delta(\mu)$ is an $N$-th order polynomial with coefficients $A_{i}$ as $\Delta(\mu)=\sum_{i} A_{i}(2 \mu)^{N-i}$. Thus the integral of eq.(9) is carried out in the bounded region where $|\Delta(\mu)| \geq 2$. If we consider complex $\mu$-plane, the Riemann sheet has a hyperelliptic structure with $N$ slits along the real axis and $N-1 \mu_{i}$ variables move between these slits. Whether $\mu_{i}$ is on the upper or lower sheet can be determined by the following formula [10].

$$
a_{1}^{2}=-2^{-N} \sum_{i=1}^{N-1} \frac{y^{ \pm}\left(\mu_{i}\right)}{P^{\prime}\left(\mu_{i}\right)}
$$

where

$$
P(\lambda)=\prod_{i=1}^{N-1}\left(\lambda-\mu_{i}\right), \quad y^{ \pm}\left(\mu_{i}\right)=\frac{1}{2}\left(\Delta\left(\mu_{i}\right) \pm\left(\Delta\left(\mu_{i}\right)^{2}-4\right)^{\frac{1}{2}}\right)
$$

and the sign $+/-$ is for the upper/lower Riemann sheets respectively. The momentum 
variable $\nu_{i}$ conjugate to $\mu_{i}$ is

$$
\nu_{i}=2(-1)^{N-i} \log \left|\frac{1}{2}\left(\Delta\left(\mu_{i}\right)+\left(\Delta\left(\mu_{i}\right)^{2}-4\right)^{\frac{1}{2}}\right)\right|
$$

where the positive branch of $\sqrt{\Delta\left(\mu_{i}\right)^{2}-4}$ is on the upper Riemann sheet. For the later convenience, we will change the indices of the quantum numbers $n_{i}$ as follows

$$
\left(n_{1}, n_{2}, \cdots, n_{N-1}\right) \rightarrow\left(n_{m}, n_{m-1}, \cdots, n_{-m+1}, n_{-m}\right)
$$

where $N=2 m+1(2 m+2)$ for $N=$ odd (even). For $N=$ odd, the quantum number $n_{0}$ is missing. The variables $\left(\mu_{1}, \mu_{2}, \cdots, \mu_{N-1}\right)$ are also relabeled as $\left(\mu_{m}, \mu_{m-1}, \cdots, \mu_{-m+1}, \mu_{-m}\right)$.

In Ref.[9], we have noticed that the quantum numbers $n_{i}$ and the symmetry of the eigenfunction have a close relationship as follows. When we define the integer $N_{S}$ as

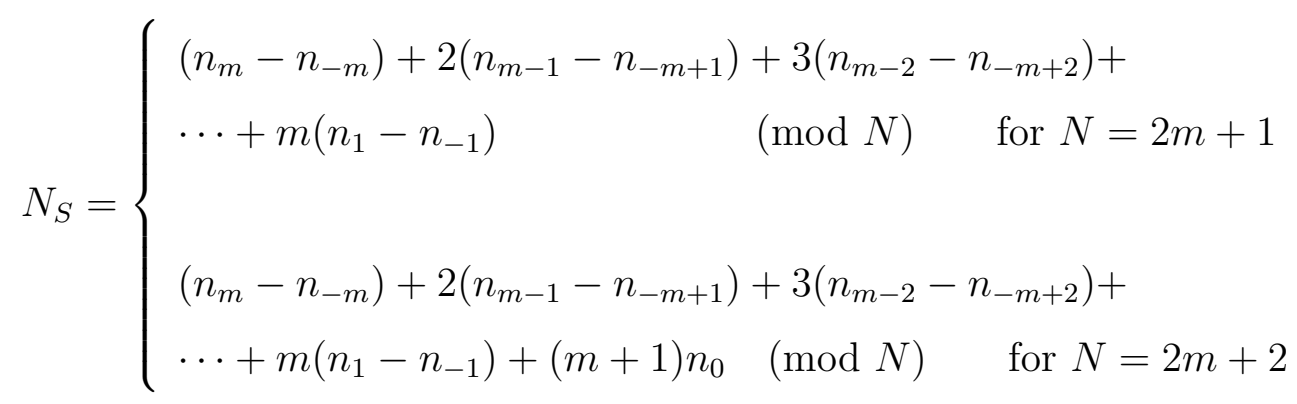

then

$$
\begin{array}{ll}
N_{S}=0 & \text { for } A \text {-symmetry } \\
N_{S}=N / 2 & \text { for } B \text {-symmetry } \\
N_{S}= \pm k \quad(1 \leq k \leq m) & \text { for } E_{k} \text {-symmetry }
\end{array}
$$

Of course, the $B$-symmetry is missing in the case of $N=$ odd.

The semiclassical wave function which is consistent with the EBK quantization is given by $($ setting $\hbar=1)[11]$

$$
\phi(\boldsymbol{q})=A(\boldsymbol{q}, \boldsymbol{I}) \exp (i S(\boldsymbol{q}, \boldsymbol{I}))
$$

where $\boldsymbol{I}=\left(I_{1}, I_{2}, \cdots, I_{N-1}\right)$ are action variables. The phase function $S(\boldsymbol{q}, \boldsymbol{I})$ is a solution of the Hamilton -Jacobi equation and also a generating function of the canonical transformation for $(\boldsymbol{p}, \boldsymbol{q}) \rightarrow(\boldsymbol{I}, \boldsymbol{\varphi})$, i.e.

$$
\boldsymbol{p} \cdot d \boldsymbol{q}+\boldsymbol{\varphi} \cdot d \boldsymbol{I}=d S
$$


and can be given by the integral

$$
S(\boldsymbol{q}, \boldsymbol{I})=\int_{C} \boldsymbol{p}(\boldsymbol{x}, \boldsymbol{I}) \cdot d \boldsymbol{x}
$$

where $C$ is a path from $O\left(\boldsymbol{p}_{0}, \boldsymbol{q}_{0}\right)$ to $P(\boldsymbol{p}, \boldsymbol{q})$ in the invariant torus $M_{\boldsymbol{I}}$ defined by $\boldsymbol{I}(\boldsymbol{p}, \boldsymbol{q})=$ const.. The amplitude $A(\boldsymbol{q}, \boldsymbol{I})$ is given by

$$
A^{2}(\boldsymbol{q}, \boldsymbol{I})=\left|\operatorname{det} \frac{\partial^{2} S}{\partial q_{i} \partial I_{j}}\right|
$$

The function $S(\boldsymbol{q}, \boldsymbol{I})$ is a multivalued function of $\boldsymbol{q}$ since there are several points $\boldsymbol{p}_{k}$ satisfying $\boldsymbol{I}\left(\boldsymbol{p}_{k}, \boldsymbol{q}\right)=$ const. for a fixed $\boldsymbol{q}$. Thus the wave function is given by a superposition, i.e.

$$
\phi(\boldsymbol{q})=\sum_{k} A_{k}(\boldsymbol{q}, \boldsymbol{I}) \exp \left(i S_{k}(\boldsymbol{q}, \boldsymbol{I})\right)
$$

where

$$
S_{k}(\boldsymbol{q}, \boldsymbol{I})=S\left(\boldsymbol{q}, \boldsymbol{I} ; \boldsymbol{p}_{k}\right)=\int_{C_{k}} \boldsymbol{p}(\boldsymbol{x}, \boldsymbol{I}) \cdot d \boldsymbol{x}
$$

and the integration path $C_{k}$ is connecting $O\left(\boldsymbol{p}_{0}, \boldsymbol{q}_{0}\right)$ with $P_{k}\left(\boldsymbol{p}_{k}, \boldsymbol{q}\right)$ and $A_{k}^{2}(\boldsymbol{q}, \boldsymbol{I})=$ $\left|\operatorname{det} \frac{\partial^{2} S_{k}}{\partial q_{i} \partial I_{j}}\right|$. The function $S_{k}(\boldsymbol{q}, \boldsymbol{I})$ is defined on the each piece of the phase space and it is continuous and finite. However, there is a phase loss $\pi / 2$ at each transition from one piece to another $[2]$.

The conserved quantities $A_{i}$ defined by eq.(4) are functions of $(\boldsymbol{p}, \boldsymbol{q})$ and they have the properties $A_{i}(s \boldsymbol{p}, s \boldsymbol{q})=A_{i}(\boldsymbol{p}, \boldsymbol{q}), A_{i}(-t \boldsymbol{p}, t \boldsymbol{q})=A_{i}(\boldsymbol{p}, \boldsymbol{q})$. Therefore the action variables $I_{i}$ also satisfy $I_{i}(s \boldsymbol{p}, s \boldsymbol{q})=I_{i}(\boldsymbol{p}, \boldsymbol{q}), I_{i}(-t \boldsymbol{p}, t \boldsymbol{q})=I_{i}(\boldsymbol{p}, \boldsymbol{q})$ and thus the shifted coordinates $(s \boldsymbol{p}, s \boldsymbol{q}),(-t \boldsymbol{p}, t \boldsymbol{q})$ stay on the invariant torus $M_{\boldsymbol{I}}$. When the shift operators $s$ and $t$ are applied to the wave function $\phi(\boldsymbol{q})$, transformations of the each component of eq.(18) are the same and it is sufficient to consider one of the components. Thus, a suffix $k$ of eqs. $(18,19)$ is dropped hereafter.

æ

Let us firstly apply the $s$ operator to $\phi(\boldsymbol{q})$, i.e.

$$
s \phi(\boldsymbol{q})=A(s \boldsymbol{q}, \boldsymbol{I}) \exp (i S(s \boldsymbol{q}, \boldsymbol{I} ; s \boldsymbol{p}))
$$


and the phase is

$$
\begin{aligned}
S(s \boldsymbol{q}, \boldsymbol{I} ; s \boldsymbol{p}) & =\int_{\left(\boldsymbol{p}_{0}, \boldsymbol{q}_{0}\right)}^{(s \boldsymbol{p}, s \boldsymbol{q})} \boldsymbol{p}(\boldsymbol{x}, \boldsymbol{I}) \cdot d \boldsymbol{x} \\
& =\int_{\left(\boldsymbol{p}_{0}, \boldsymbol{q}_{0}\right)}^{(\boldsymbol{p}, \boldsymbol{q})} \boldsymbol{p} \cdot d \boldsymbol{x}+\int_{(\boldsymbol{p}, \boldsymbol{q})}^{(s \boldsymbol{p}, s \boldsymbol{q})} \boldsymbol{p} \cdot d \boldsymbol{x}+\oint \boldsymbol{p} \cdot d \boldsymbol{x} \\
& =S(\boldsymbol{q}, \boldsymbol{I})+\int_{(\boldsymbol{p}, \boldsymbol{q})}^{(s \boldsymbol{p}, s \boldsymbol{q})} \boldsymbol{p} \cdot d \boldsymbol{x}+\sum_{i} l_{i} I_{i}
\end{aligned}
$$

The term $\oint \boldsymbol{p} \cdot d \boldsymbol{x}$ is necessary since we do not specify an integration path and it becomes

$$
\oint \boldsymbol{p}(\boldsymbol{x}, \boldsymbol{I}) \cdot d \boldsymbol{x}=\oint \boldsymbol{\nu}(\boldsymbol{\mu}, \boldsymbol{I}) \cdot d \boldsymbol{\mu}=\sum_{i} l_{i} I_{i} \quad\left(l_{i} ; \text { integer }\right)
$$

since the phase space can be mapped to $(\boldsymbol{\nu}, \boldsymbol{\mu})$-space by the canonical transformation. When the path $(\boldsymbol{p}, \boldsymbol{q}) \rightarrow(s \boldsymbol{p}, s \boldsymbol{q})$ is mapped to $(\boldsymbol{\nu}, \boldsymbol{\mu})$-space, it winds the $\mu_{i}$-interval several times. So we will define the minimum path in $(\boldsymbol{\nu}, \boldsymbol{\mu})$-space such that the variation of the angle variable $\varphi_{i}$ is $0 \leq \Delta \varphi_{i}<2 \pi$. If we denote the corresponding minimum path $(\boldsymbol{p}, \boldsymbol{q}) \rightarrow(s \boldsymbol{p}, s \boldsymbol{q})$ in $(\boldsymbol{p}, \boldsymbol{q})$-space as $C_{1}$, then

$$
\int_{(\boldsymbol{p}, \boldsymbol{q})}^{(s \boldsymbol{p}, s \boldsymbol{q})} \boldsymbol{p} \cdot d \boldsymbol{x}=\int_{C_{1}} \boldsymbol{p} \cdot d \boldsymbol{x}+\sum_{i} l_{i}^{\prime} I_{i} \quad\left(l_{i}^{\prime} ; \text { integer }\right)
$$

Now the proplem is how to calculate $\int_{C_{1}} \boldsymbol{p} \cdot d \boldsymbol{x}$. Let us define the path $C_{2}$ by applying the $s$ operator $C_{2}=s C_{1}$, i.e.

$$
(\boldsymbol{p}, \boldsymbol{q}) \in C_{1}, \quad\left(\boldsymbol{p}^{\prime}, \boldsymbol{q}^{\prime}\right) \in C_{2}, \quad \boldsymbol{p}^{\prime}\left(\boldsymbol{q}^{\prime}, \boldsymbol{I}\right)=\boldsymbol{p}^{\prime}(s \boldsymbol{q}, \boldsymbol{I})=s \boldsymbol{p}(\boldsymbol{q}, \boldsymbol{I})
$$

Then

$$
\begin{aligned}
\int_{C_{2}} \boldsymbol{p}^{\prime}\left(\boldsymbol{x}^{\prime}, \boldsymbol{I}\right) \cdot d \boldsymbol{x}^{\prime} & =\int_{C_{1}} \boldsymbol{p}^{\prime}(s \boldsymbol{x}, \boldsymbol{I}) \cdot d(s \boldsymbol{x}) \\
& =\int_{C_{1}} s \boldsymbol{p}(\boldsymbol{x}, \boldsymbol{I}) \cdot d(s \boldsymbol{x}) \\
& =\int_{C_{1}} \boldsymbol{p}(\boldsymbol{x}, \boldsymbol{I}) \cdot d \boldsymbol{x}
\end{aligned}
$$

since ${ }^{T} s s=1$ in a matrix representation. Therefore

$$
S\left(s^{2} \boldsymbol{q}, \boldsymbol{I}\right)-S(s \boldsymbol{q}, \boldsymbol{I})=S(s \boldsymbol{q}, \boldsymbol{I})-S(\boldsymbol{q}, \boldsymbol{I})
$$

Making use of $\varphi_{i}=\frac{\partial S}{\partial I_{i}}$, one can get the variation of the angle $\varphi_{i}$ as

$$
\varphi_{i}\left(s^{2} \boldsymbol{q}, \boldsymbol{I}\right)-\varphi_{i}(s \boldsymbol{q}, \boldsymbol{I})=\varphi_{i}(s \boldsymbol{q}, \boldsymbol{I})-\varphi_{i}(\boldsymbol{q}, \boldsymbol{I})
$$


Thus

$$
\Delta \varphi_{i}\left(C_{2}\right)=\Delta \varphi_{i}\left(C_{1}\right)
$$

Since $C_{1}$ is a minimum path $\left(0 \leq \Delta \varphi_{i}\left(C_{1}\right)<2 \pi\right), C_{2}$ is also a minimum path $\left(0 \leq \Delta \varphi_{i}\left(C_{2}\right)<\right.$ $2 \pi)$ connecting $(s \boldsymbol{p}, s \boldsymbol{q}) \rightarrow\left(s^{2} \boldsymbol{p}, s^{2} \boldsymbol{q}\right)$. In the same way

$$
\begin{gathered}
\int_{C_{1}} \boldsymbol{p} \cdot d \boldsymbol{x}=\int_{s C_{1}} \boldsymbol{p} \cdot d \boldsymbol{x}=\int_{s^{2} C_{1}} \boldsymbol{p} \cdot d \boldsymbol{x}=\cdots=\int_{s^{N-1} C_{1}} \boldsymbol{p} \cdot d \boldsymbol{x} \\
\int_{C_{1}} \boldsymbol{p} \cdot d \boldsymbol{x}=\frac{1}{N} \int_{C_{1}+s C_{1}+\cdots+s^{N-1} C_{1}} \boldsymbol{p} \cdot d \boldsymbol{x}=\frac{1}{N} \oint_{C} \boldsymbol{p} \cdot d \boldsymbol{x}
\end{gathered}
$$

where $C=C_{1}+s C_{1}+\cdots+s^{N-1} C_{1}$ and $C$ is a closed path.

Let us evaluate $\oint_{C} \boldsymbol{p} \cdot d \boldsymbol{x}$ by mapping $C$ to $(\boldsymbol{\nu}, \boldsymbol{\mu})$-space, i.e.

$$
\oint_{C} \boldsymbol{p} \cdot d \boldsymbol{x}=\oint \boldsymbol{\nu} \cdot d \boldsymbol{\mu}=\sum_{i=-m}^{m} k_{i} I_{i}
$$

where $k_{i}$ is a winding number of the variable $\mu_{i}$ in the $i$-th interval. In order to obtain $k_{i}$, we have carried out numerical calculation. Firstly, we take an arbitrary point $P_{1}=(\boldsymbol{p}, \boldsymbol{q})$ is the phase space. This point $P_{1}$ determines the conserved quantities $I_{i}$ and, thus, the invariant torus. The closed path consists of $P_{1} \rightarrow P_{2} \rightarrow \cdots \rightarrow P_{N} \rightarrow P_{N+1}=P_{1}$ where $P_{k}=s^{k-1} P_{1}=\left(s^{k-1} \boldsymbol{p}, s^{k-1} \boldsymbol{q}\right)$. Secondly, the point $P_{k}$ is mapped to $(\boldsymbol{\nu}, \boldsymbol{\mu})$-space and a number of winding is counted for each $\mu_{i}$. Since the path $P_{k} \rightarrow P_{k+1}$ is a minimum one, the winding number is uniquely determined. We have done the calculation by taking thousands of random points $P_{1}$ for $N=3,4,5$ and 6 . The typical cases for $N=5$ and 6 are shown in Figs.1 and 2. In any case, the winding numbers which are obtained by the numerical calculation are $(1,1),(-1,2,1),(1,-2,-2,1)$ and $(-1,2,3,-2,1)$ for $N=3,4,5$ and 6 respectively. (the increasing direction of the upper Riemann sheet is defined as a positive rotation and the sequence is defined as $\left.\mu_{m}, \mu_{m-1}, \cdots, \mu_{-m+1}, \mu_{-m}\right)$ When we consider a closed path $C$ in the complex $\mu$-plane, the positive branch of $\sqrt{\Delta\left(\mu_{i}\right)^{2}-4}$ is on the upper Riemann sheet. In order to get a correct sign of $\nu_{i}$, we should take into account the phase $(-1)^{N-i}$ (eq.(12)) and $k_{i}$ can be finally given by

$$
k_{i}=\operatorname{sign}(i)(m+1-|i|) \quad(-m \leq i \leq m)
$$


and this is our main result. Although eq.(32) is obtained by the numerical calculation up to $N=6$, we will conjecture that it will hold generally for the $N$-particle case and we hope it will be proved by more mathematical way. Using eq.(32), eq.(31) becomes

$$
\oint \boldsymbol{\nu} \cdot d \boldsymbol{\mu}= \begin{cases}\sum_{i=1}^{m}(m+1-i)\left(I_{i}-I_{-i}\right), & \text { for } N=2 m+1 \\ \sum_{i=1}^{m}(m+1-i)\left(I_{i}-I_{-i}\right)+(m+1) I_{0}, & \text { for } N=2 m+2\end{cases}
$$

When the EBK quantization $I_{i} \rightarrow 2 \pi\left(n_{i}+\frac{1}{2}\right)$ is applied, the term $2 \pi \times 1 / 2=\pi$ coming from the Maslov index is canceled by the phase loss of each two turning points of the invariant torus. Finally one can get

$$
\frac{1}{2 \pi} \oint \boldsymbol{\nu} \cdot d \boldsymbol{\mu}= \begin{cases}\sum_{i=1}^{m}(m+1-i)\left(n_{i}-n_{-i}\right), & \text { for } N=2 m+1 \\ \sum_{i=1}^{m}(m+1-i)\left(n_{i}-n_{-i}\right)+(m+1) n_{0}, & \text { for } N=2 m+2\end{cases}
$$

Using the definition of $N_{S}$ (eq.(13))

$$
\int_{C_{1}} \boldsymbol{p} \cdot d \boldsymbol{x}=\frac{1}{N} \oint \boldsymbol{\nu} \cdot d \boldsymbol{\mu}=2 \pi \frac{N_{S}}{N}+2 \pi n_{s} \quad\left(n_{s} ; \text { integer }\right)
$$

Therefore

$$
S(s \boldsymbol{q}, \boldsymbol{I} ; s \boldsymbol{p})=S(\boldsymbol{q}, \boldsymbol{I} ; \boldsymbol{p})+2 \pi \frac{N_{S}}{N}+2 \pi n_{s}+\sum_{i}\left(l_{i}+l_{i}^{\prime}\right) I_{i}
$$

The substitution $I_{i}=2 \pi\left(n_{i}+\frac{1}{2}\right)$ and the subtraction of the phase loss $\left(l_{i}+l_{i}^{\prime}\right) \pi$ give

$$
S(s \boldsymbol{q}, \boldsymbol{I} ; s \boldsymbol{p})=S(\boldsymbol{q}, \boldsymbol{I} ; \boldsymbol{p})+2 \pi \frac{N_{S}}{N}+2 \pi n_{s}+\sum_{i} 2 \pi\left(l_{i}+l_{i}^{\prime}\right) n_{i}
$$

and finally

$$
\begin{aligned}
\exp (i S(s \boldsymbol{q}, \boldsymbol{I} ; s \boldsymbol{p})) & =\exp (i S(\boldsymbol{q}, \boldsymbol{I} ; \boldsymbol{p})) \exp \left(2 \pi i \frac{N_{S}}{N}\right) \\
& =\zeta^{l} \exp (i S(\boldsymbol{q}, \boldsymbol{I} ; \boldsymbol{p}))
\end{aligned}
$$

when $N_{S}=l$. Eq.(38) exactly corresponds to eq.(7). Concerning the amplitude $A(\boldsymbol{q}, \boldsymbol{I})$, one can easily see that $A(\boldsymbol{q}, \boldsymbol{I})=A(s \boldsymbol{q}, \boldsymbol{I})$ because of eq.(36). 
Secondly we will consider the $t$ operator. To be consistent with eq.(8), $t \phi(\boldsymbol{q})=\phi(\boldsymbol{q})^{*}$, which determines the phase, the initial point $O\left(\boldsymbol{p}_{0}, \boldsymbol{q}_{0}\right)$ of the path $C$ should be chosen such that $t O=\left(-t \boldsymbol{p}_{0}, t \boldsymbol{q}_{0}\right)=\left(\boldsymbol{p}_{0}, \boldsymbol{q}_{0}\right)=O$. Since the invariant torus has the property $\boldsymbol{I}(-t \boldsymbol{p}, t \boldsymbol{q})=\boldsymbol{I}(\boldsymbol{p}, \boldsymbol{q})$, the transformation of $\phi(\boldsymbol{q})$ becomes

$$
\begin{gathered}
t \phi(\boldsymbol{q})=A(t \boldsymbol{q}, \boldsymbol{I}) \exp (i S(t \boldsymbol{q}, \boldsymbol{I} ;-t \boldsymbol{p})) \\
S(t \boldsymbol{q}, \boldsymbol{I} ;-t \boldsymbol{p})=\int_{\left(\boldsymbol{p}_{0}, \boldsymbol{q}_{0}\right)}^{(-t \boldsymbol{p}, t \boldsymbol{q})} \boldsymbol{p}(\boldsymbol{x}, \boldsymbol{I}) \cdot d \boldsymbol{x}
\end{gathered}
$$

Let us define a path $C^{\prime}$ on the invariant torus by applying the $t$ operator $C^{\prime}=t C$, i.e.

$$
(\boldsymbol{p}, \boldsymbol{q}) \in C, \quad\left(\boldsymbol{p}^{\prime}, \boldsymbol{q}^{\prime}\right) \in C^{\prime}, \quad \boldsymbol{p}^{\prime}\left(\boldsymbol{q}^{\prime}, \boldsymbol{I}\right)=\boldsymbol{p}^{\prime}(t \boldsymbol{q}, \boldsymbol{I})=-t \boldsymbol{p}(\boldsymbol{q}, \boldsymbol{I})
$$

Thus

$$
\begin{aligned}
S(t \boldsymbol{q}, \boldsymbol{I} ;-t \boldsymbol{p}) & =\int_{C^{\prime}} \boldsymbol{p}^{\prime}\left(\boldsymbol{x}^{\prime}, \boldsymbol{I}\right) \cdot d \boldsymbol{x}^{\prime} \\
& =-\int_{C} t \boldsymbol{p}(\boldsymbol{x}, \boldsymbol{I}) \cdot d(t \boldsymbol{x})=-\int_{C} \boldsymbol{p}(\boldsymbol{x}, \boldsymbol{I}) \cdot d(t \boldsymbol{I}) \cdot d \boldsymbol{x} \\
& =-S(\boldsymbol{q}, \boldsymbol{I} ; \boldsymbol{p})
\end{aligned}
$$

since ${ }^{T} t t=1$, and $A(t \boldsymbol{q}, \boldsymbol{I})=A(\boldsymbol{q}, \boldsymbol{I})$. Finally one can get $t \phi(\boldsymbol{q})=\phi(\boldsymbol{q})^{*}$ and show that $\phi(\boldsymbol{q})$ satisfies eq. (8).

In summary, we have considered the symmetry of the eigenfunction of the periodic Toda lattice in the semiclassical region by the EBK quantization and found that the integer quantum numbers have a close relationship to a structure of the invariant torus of the classical motion. However, the symmetric property is a discrete one and it does not depend on the excitation energy or the size of $\hbar$. Therefore it is concluded that this relationship is one of the distinctive features of the periodic Toda lattice. $æ$ 


\section{References}

[1] M. Toda, J.Phys.Soc.Japan 22 (1969) 431; Theory of nonlinear lattices (Springer, Berlin, 1981)

[2] J.B. Keller, Ann.Phys. (NY) 4 (1958) 180

[3] M.C. Gutzwiller, Ann.Phys. (NY) 124 (1980) 347; 133 (1981) 304

[4] E.K. Sklyanin, Nonlinear equations in classical and quantum field theory, Lecture Notes in Physics, Vol.226 (Springer, Berlin, 1985)

[5] M. Fowler and H. Frahm, Phys.Rev. B39 (1989) 11800

[6] S. Isola, H. Kantz and R. Livi, J.Phys. A24 (1991) 3061

[7] A. Matsuyama, Phys.Lett. A161 (1991) 124

[8] V. Pasquier and M. Gaudin, J.Phys. A25 (1992) 5243

[9] A. Matsuyama, Ann.Phys. (NY) 220 (1992) 300

[10] P. van Moerbeke, Invent. Math. 37 (1976) 45

[11] A.M. Ozorio de Almeida, Hamiltonian systems: chaos and quantization (Cambridge University Press, Cambridge, 1988)

$æ$ 


\section{Table and Figure captions}

Table 1 Eigenfunctions and the matrix elements of the conserved quantities $A_{i}$ for each symmetry in the non-degenerate (A) / degenerate (B) case.

Fig.1 Behavior of auxiliary spectra $\mu_{i}$ for $N=5$. The point $P_{1}$ is $\boldsymbol{p}=(-0.6,-0.4,0.2$, $0.3,0.5)$ and $\boldsymbol{q}=(-0.6,-0.4,0.0,0.2,0.8)$. Dots $(\bullet)$ and triangles $(\triangle)$ show the points of the upper and lower Riemann sheets respectively. Solid (dashed) lines show the movements of $\mu_{i}$ in the upper (lower) Riemann sheet.

Fig.2 Behavior of auxiliary spectra $\mu_{i}$ for $N=6$. The point $P_{1}$ is $\boldsymbol{p}=(-0.5,-0.4,-0.1$, $0.2,0.3,0.5)$ and $\boldsymbol{q}=(-0.6,-0.4,0.0,0.1,0.3,0.6)$. Descriptions are the same as Fig.1.

$æ$ 
Table 1A

\begin{tabular}{|c|c|c|c|}
\hline sym. & w.f. & $\mathrm{A}_{\text {odd }}$ & $\mathrm{A}_{\text {even }}$ \\
\hline$A$ & $\phi^{A_{1}}$ & 0 & $\left\langle\phi^{A_{1}}\left|A_{i}\right| \phi^{A_{1}}\right\rangle$ \\
$B$ & $\phi^{B_{1}}$ & 0 & $\left\langle\phi^{B_{1}}\left|A_{i}\right| \phi^{B_{1}}\right\rangle$ \\
\hline
\end{tabular}

Table 1B

\begin{tabular}{|c|c|c|c|}
\hline sym. & w.f. & $\mathrm{A}_{\text {odd }}$ & $\mathrm{A}_{\text {even }}$ \\
\hline$A$ & $\frac{1}{\sqrt{2}}\left(\phi^{A_{1}} \pm i \phi^{A_{2}}\right)$ & $\pm \operatorname{Im}\left\langle\phi^{A_{2}}\left|A_{i}\right| \phi^{A_{1}}\right\rangle$ & $\frac{1}{2}\left(\left\langle\phi^{A_{1}}\left|A_{i}\right| \phi^{A_{1}}\right\rangle+\left\langle\phi^{A_{2}}\left|A_{i}\right| \phi^{A_{2}}\right\rangle\right)$ \\
$B$ & $\frac{1}{\sqrt{2}}\left(\phi^{B_{1}} \pm i \phi^{B_{2}}\right)$ & $\pm \operatorname{Im}\left\langle\phi^{B_{2}}\left|A_{i}\right| \phi^{B_{1}}\right\rangle$ & $\frac{1}{2}\left(\left\langle\phi^{B_{1}}\left|A_{i}\right| \phi^{B_{1}}\right\rangle+\left\langle\phi^{B_{2}}\left|A_{i}\right| \phi^{B_{2}}\right\rangle\right)$ \\
$E$ & $\phi^{E}, \quad \phi^{E^{*}}$ & $\pm\left\langle\phi^{E}\left|A_{i}\right| \phi^{E}\right\rangle$ & $\left\langle\phi^{E}\left|A_{i}\right| \phi^{E}\right\rangle$ \\
\hdashline & & \\
\hline
\end{tabular}

